\title{
Orthopädisch-unfallchirurgische Forschung in Deutschland
}

\author{
Research on Orthopaedic and Casualty Surgery in Germany
}

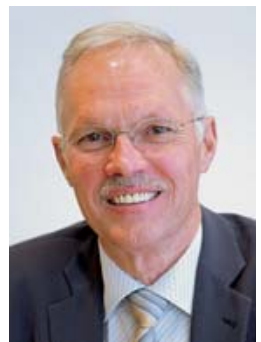

F. U. Niethard

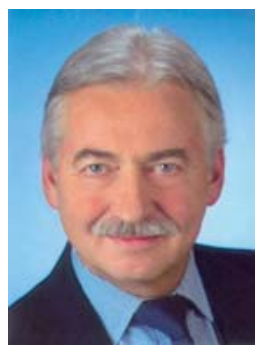

K. Weise

Bibliografie

DOI $10.1055 / \mathrm{s}-0030-1249791$ Z Orthop Unfall 2010; 148: 143144 ๑ Georg Thieme Verlag KG Stuttgart . New York. ISSN 1864-6697

\section{Korrespondenzadressen}

Prof. Dr. med. Fritz U. Niethard Orthopädische Klinik

Universitätsklinik der RWTH Aachen

Pauwelsstraße 30

52074 Aachen

Tel.: 0241/808-94 10

Fax: 0241/808-2453

funiethard@

orthopaedie-aachen.de

Prof. Dr. med. Kuno Weise BG-Unfallklinik

Schnarrenbergstraße 95 72076 Tübingen

Tel.: 0 7071/606-1001

Fax: 07071/606-1002

weise@bgu-tuebingen.de
Orthopädie und Unfallchirurgie ist ein großes Fach mit über 13000 berufstätigen Ärzten. Diese sind in ihrem alltäglichen Schaffen einem wissenschaftlichen Standard verpflichtet, der früher fast ausschließlich in den Universitätsklinika erarbeitet wurde. Viele bahnbrechende Erfolge in Orthopädie und Unfallchirurgie sind von Pionieren erzielt worden, die wagemutig große Schritte in ein bis dahin „unbeackertes“ Land gesetzt haben. Zahlreiche Operationsmethoden (Osteosynthese, Endoprothetik) haben so ihren Siegeszug durch die Welt angetreten. Manche sind aber auch gescheitert und haben sich nicht durchsetzen können.

Derartige häufig auf Einzelfallbeschreibungen basierende innovative Ansätze sind heute kaum mehr denkbar. Mehr und mehr geht es um vergleichende Fragestellungen, bei denen nicht nur das „Wie“, sondern auch das „Wann“, das „Wie effizient“ und sogar das „Wie teuer“ im Vordergrund steht. Dafür ist der Aufbau einer Infrastruktur erforderlich, die in der Regel nicht mehr allein von einer Universitätsklinik geschultert werden kann. Dennoch wird an vielen Universitätsstandorten Forschung in Orthopädie und Unfallchirurgie nicht als Aufgabe des Faches, sondern als Aufgabe des Standorts und als Wettbewerb gegenüber anderen Universitätsklinika gesehen. So erklärt es sich, dass die heute unerlässlichen randomisierten Studien in Orthopädie und Unfallchirurgie nur selten anzutreffen sind. Otto et al. [1] führen in einer Veröffentlichung in dieser Zeitschrift aus, dass Arbeiten mit orthopädischem Inhalt nur 0,2\% aller klinischen Studien und 0,5\% der randomisierten kontrollierten Studien darstellen. Offenbar ein Beleg für das Postulat, wonach in allen chirurgischen Fächern zu wenig geforscht wird.

Sind Chirurgen und darunter Orthopäden und Unfallchirurgen etwa keine Forscher oder wo liegt der Fehler? Bernstein und Mitarbeiter haben eine Befragung zur Vereinbarkeit von klinischer und akademischer Entwicklung an orthopädischen und unfallchirurgischen Universitätskliniken in Deutschland durchgeführt. Mittels einer Fragebogenaktion bei 105 an Universitätsklinika tätigen Ärzten wurde der Konflikt zwischen Forschung, klinischer Tätigkeit und familiären Verpflichtungen deutlich: In diesem Spannungsfeld hat die Tätigkeit in der Klinik, d.h. vor allem eine hochwertige Weiterbildung, eindeutigen Vorrang und dies noch vor dem Privatleben ([2], Tab. 1).
Der Qualität der Weiterbildung wird deswegen ein hoher Stellenwert eingeräumt, weil sich nach allgemeiner Meinung danach auch die Karrierechancen und die Perspektiven im Beruf bemessen lassen. Die Mehrheit ist davon überzeugt, dass eher die klinische als die Forschungsleistung bestimmend für das berufliche Schicksal sein wird. Dafür sind die Ärzte sogar bereit, auf Geld zu verzichten, denn das Gehalt rangiert erst an letzter Stelle der Prioritätenliste. Davor wird der Forschungsauftrag genannt, dem man gerne eine höhere Priorität beimessen würde. So sind nur 55\% mit der eigenen Forschungsleistung zufrieden, $39 \%$ der Befragten eher unzufrieden.

Was also gilt es zu tun? Die Deutsche Gesellschaft für Orthopädie und Orthopädische Chirurgie hat bereits vor mehreren Jahren erkannt, dass vor allem in der Grundlagenforschung Qualität nicht mehr im Alleingang, sondern durch Netzwerkbildung erreicht wird. Durch eine Anschubfinanzierung wurde zur Bildung von Netzwerkstrukturen angeregt, die sich mittlerweile fest etabliert haben. So konnten aus dem Netzwerk „Regenerative Knorpeltherapien“ mehrere DFG-geförderte Projekte weiterentwickelt werden. Das Netzwerk „Biomechanik“ wiederum hat inzwischen eine Drittmittelförderung von über 6000000,-EUR für weiterführende Projekte erhalten.

Alle diese Forschungsprojekte sind jedoch nicht mehr von Ärzten und Oberärzten in der Klinik zu leisten. Sie können nur noch von Grundlagenforschern erbracht werden, die eng mit den Kliniken kooperieren. Ziel der Fachgesellschaften muss es daher sein, weitere Strukturen aufzubauen, die die zukunftsträchtigen Forschungsfelder für das Fach Orthopädie und Unfallchirurgie bearbeiten. Dazu gehört auch die mesenchymale Stammzelltherapie. Anlässlich eines Expertenworkshops der Deutschen Gesellschaft für Orthopädie und Unfallchirurgie wurde die Datenlage zum Einsatz von MSC im experimentellen und klinischen Bereich zusammengefasst und das Potenzial zur künftigen Durchführung klinischer Studien geprüft [3]. Daraus wurde abgeleitet, dass weitere klinische Studien erforderlich sind, um das tatsächliche Potenzial der Applikation einschätzen zu können. Und hierfür wiederum ist eine verstärkte Zusammenarbeit der Forschungszentren sinnvoll. Eine Aufgabe, der sich die orthopädischen und unfallchirurgischen Universitätskliniken im Verbund annehmen müssen. Sie erfordert 
eine Schwerpunktbildung und Ausrichtung der Standorte mit Schaffung geeigneter Substrukturen. Dazu gehören nicht nur geeignete Labors, sondern auch Dauerstellen im W-Bereich.

Und welche Lösungen gibt es für die klinische Forschung? Otto et al. [1] weisen darauf hin, dass die Novellierung des Medizinproduktegesetzes einen erheblich erhöhten Bedarf an klinischer Forschung mit sich bringen wird. Diese Entwicklung antizipierend hat die Deutsche Gesellschaft für Orthopädie und Unfallchirurgie bereits Kurse zur Zertifizierung orthopädisch-unfallchirurgischer Prüfärzte eingerichtet [4]. Allerdings ist damit das Spannungsfeld zwischen Forschungstätigkeit, klinischen Aufgaben und Familienhintergrund noch nicht abgeglichen. Es bedarf auch für die klinische Forschung geeigneter Substrukturen, um über eine Netzwerkbildung geeignete Studienprotokolle aufbauen und qualifizierte Aussagen treffen zu können. Die Deutsche Gesellschaft für Chirurgie hat mit der Einrichtung eines Studienzentrums in Heidelberg einen großen Schritt in diese Richtung gemacht und damit breite Anerkennung gefunden. Dieses Studienzentrum steht auch für die Mitglieder der chirurgischen Fachgesellschaften offen [1]. Die Deutsche Gesellschaft für Orthopädie und Unfallchirurgie wird sich auch dieses Themas annehmen, um die Breite des Faches von der Grundlagen- bis zur Versorgungsforschung darstellen, fördern und weiterentwickeln zu können.

\section{F. U. Niethard, Aachen \\ K. Weise, Tübingen}

\section{Literatur}

1 Otto C, Siewe J, Zarghooni K et al. Klinische Forschung in der Orthopädie - Aufbau eines Studienzentrums in der Orthopädie/Unfallchirurgie. Z Orthop Unfall 2010; 148: 145-148

2 Bernstein P, Stiehler M, Haasper C. Ergebnisse einer Befragung zur Vereinbarkeit von klinischer und akademischer Entwicklung an orthopädischen und unfallchirurgischen Universitätskliniken in Deutschland. Z Orthop Unfall 2010; 148: 155-162

3 Kasten P, Bernstein P, Biewener A et al. Perspektiven klinischer mesenchymaler Stammzelltherapie bei muskuloskeletalen Erkrankungen in Deutschland. Z Orthop Unfall 2010; 148: 149-154

4 Günther KP, Zwipp H. Orthopädisch-unfallchirurgische Forschung: Woher, wo, wohin? Z Orthop Unfall 2009; 147: 539-541 